\title{
Total claviculectomy in Ewing's sarcoma clavicle: A rare case report and review of literature
}

\author{
Manoranjan Mallik ${ }^{1}$, S. S Nayak ${ }^{2 *}$, Sandeep Lenka ${ }^{3}$, Nihar Ranjan Mishra ${ }^{4}$, Amit Das ${ }^{5}$ \\ ${ }^{1}$ Associate Professor, ${ }^{2-4}$ Senior Resident, ${ }^{5}$ Junior Resident, Dept. of Orthopaedics, SCB Medical College and Hospital, Cuttack, Odisha, \\ India
}

*Corresponding Author: S. S Nayak

Email: nayak.subhranshu@gmail.com

\begin{abstract}
Ewing's sarcoma rarely arises from clavicle. Total claviculectomy is a generally accepted method of treatment with good functional outcome. We report a case of a 15 year male patient diagnosed with Ewing's sarcoma clavicle treated with total claviculectomy. The purpose of our study is to evaluate the functional and oncological outcome after total resection of clavicle. This patient had full range of motion of the shoulder joint without any deficit. Thus, total claviculectomy can be considered as procedure in primary clavicular malignancy.
\end{abstract}

Keywords: Total claviculectomy, Functional outcome, Ewing's sarcoma.

\section{Introduction}

Ewing sarcoma is the third most common non-hematologic primary bone malignancy, the second most common in patients younger than 30 years of age and the most common in patients younger than 10 years of age. ${ }^{1}$ It belongs to a small round cell tumour arising most commonly from metaphysis of long bone (extending to diaphysis) \& flat bones such as pelvic and pectoral bones. Ewing sarcoma arising from clavicle is very rare with incidence of less than $1 \%$ of all bone tumours ${ }^{2} \&$ there are few reports in the literature about the appropriate management. Total claviculectomy is a wellestablished method for management of clavicle Ewing sarcoma. ${ }^{3-4}$ Clavicle can be resected totally without need for reconstruction.

\section{Case Study}

We report a case of Ewing sarcoma of right clavicle in a 15 year old male patient presenting to our OPD. He had a history of trauma 2 years back immediately after which he developed swelling \& erythema over right clavicle \& was unable to move his right shoulder \& was treated else-where with bracing for fracture of clavicle (Fig. 1c). Pain was subsided with medications, he was able to move the shoulder after 1 month, but the swelling gradually increased. There was no fever or weight loss and decreased appetite. At presentation, local examination revealed a $12 \mathrm{~cm} \times 6 \mathrm{~cm}$ palpable mass over the right clavicle (Fig. 1b). It was tender on Palpation, firm in consistency. The swelling is not fixed to the skin but inseparable from underlying bone. There was no local rise of temperature and erythema. Smooth and well defined margins and there were no venous engorgement, scar marks, sinus or fistula. The patient had full shoulder range of motion (ROM), with normal strength, and no neurovascular deficit.

1. Radiographs of right shoulder with clavicle demonstrated an expansile lytic bony lesion involving the right clavicle except the lateral end with cortical irregularities \&ill-defined medial end with abrupt change in width of the bone in the lateral aspect of clavicle (Fig. 1c).
2. Laboratory investigation revealed TLC of 10,000/cubic $\mathrm{mm}$, Erythrocyte sedimentation rate of $19 \mathrm{~mm} / \mathrm{h}$, Alkaline phosphatase of $59.90 \mathrm{U} / \mathrm{L}$ and C-reaction protein $3.5 \mathrm{mg} / \mathrm{dl}$.

3. MRI study showed soft tissue mass, in-homogenously enhancing expansile mass of size $9 \times 7 \times 7 \mathrm{~cm}$ with bony lysis surrounding over right clavicle as well as marrow involvement, there was no neurovascular encasement and no extension into thoracic cavity suggestive of Malignant GCT (Fig. 1e).

4. FNAC of the swelling showed presence of small round cells arranged in loose clustures in dissociated manner with ROSSETTE like structures in background-features suggestive of small round cell tumor.

5. Tru-cut biopsy demonstrated malignant Round cell tumor consistent with PNET/EWING'S SARCOMA. HRCT of thorax showed no mass or enhancement suggestive of secondaries.

After all necessary work-ups, patient was sent for preoperative chemotherapy. Chemotherapy regimens adopted were Vincristine, Adriamycin, Cyclophosphamide, Ifosfamide, Etoposide. During chemotherapy period, the minimum white blood cells of the patient was within normal limits, and the patient exprienced severe nausea and vomiting which was obviously alleviated after treatment with metoclopramide.

After chemotherapy, proper pre-operative Aneasthetic check up was carried out. Total resection of clavicle was performed (Fig. 1d). There was no intra operative complications. The patient's blood loss was $350 \mathrm{ml}$, and he tolerated the procedure well. No violation of the tumor mass occurred. The whole clavicle with tumor mass was sent for histopathology \& Immunohistochemistry.

Biopsy of the mass were morphologically and histologically consistent with small round blue cell tumour which showed positively for mic-2 (CD99) on immunohistochemistry.

Postoperative phase was un-eventful, and the wound healed well. He received postoperative chemotherapy 
\{Vincritine, adriamycin, cyclophosphamine (VAC) alternatively with Etoposide and ifosfamide (EI) given for 5 cycles $\}$ \& radiotherapy. Postoperatively, the patient's right arm was immobilized for 3 weeks before allowing him to do supervised passive \& active shoulder physiotherapy gradually. He has received no other formal physical therapy.
Functionally, he uses his right upper extremity normally, with strength equal to the contralateral nondominant side (Fig. $2 a, b, c)$. He has intact vascular and neurological status of the right upper extremity and full range of motion of his right shoulder.

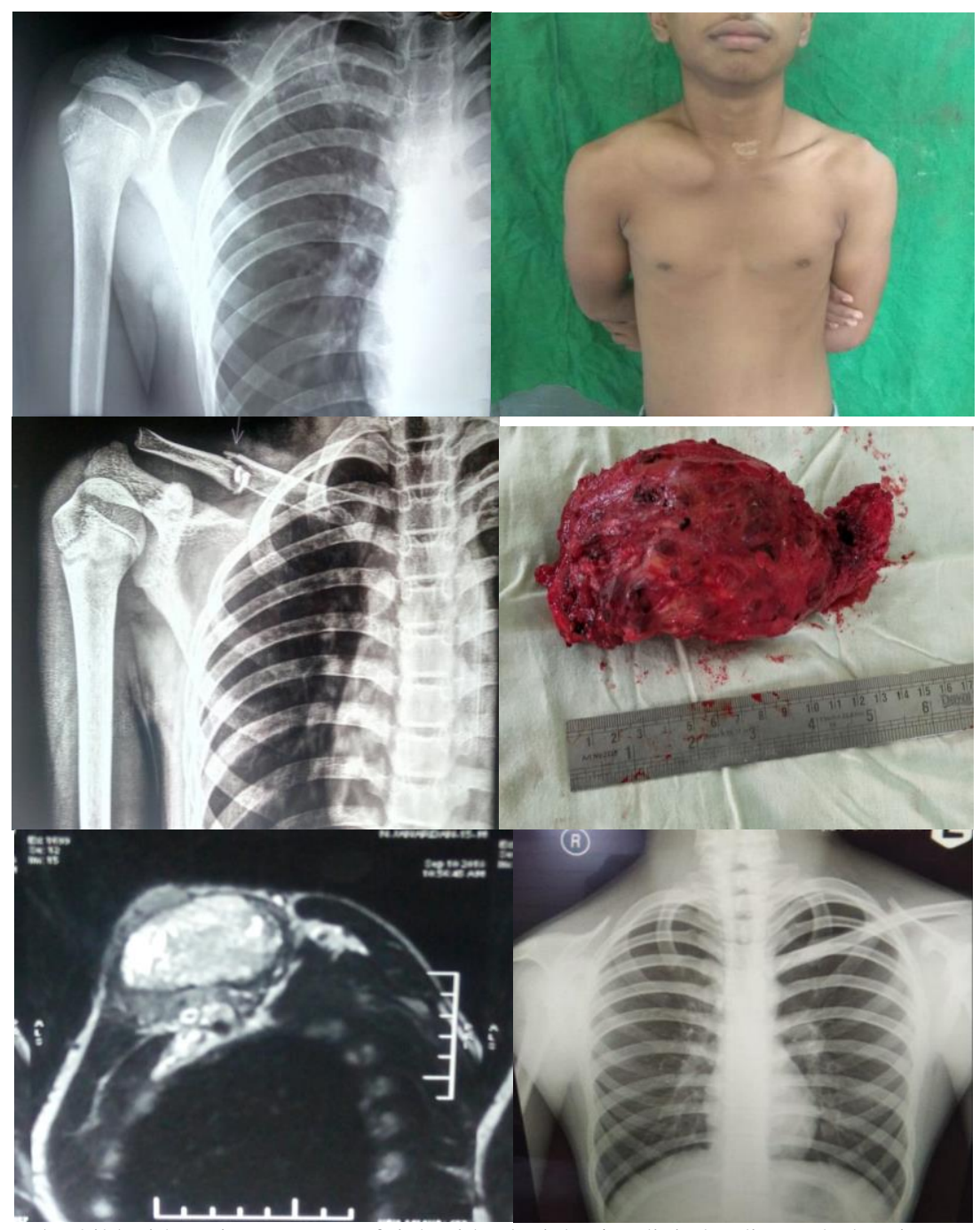

Fig 1a-f : Fifteen year old male child with Ewing sarcoma of right side clavicle. 1a: digital radiograph showing swelling of right side clavicle; 1b: clinical photograph; 1c: fracture of right side clavicle 2 years back; 1d: surgical specimen; 1e: MRI findings of the growth; 1f: postoperative radiograph showing total resection of clavicle.
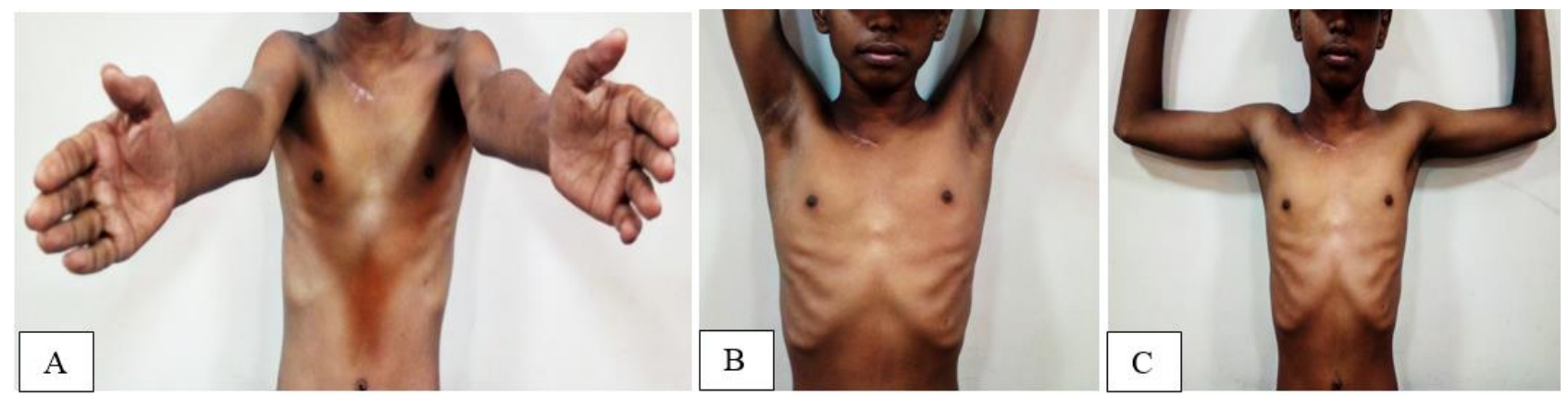

Fig. 2a-c: Post operative shoulder range of motions after 1 year. 


\section{Discussion}

Ewing sarcoma is second most common malignant bone tumour occurring in late childhood and adults (Approx 1\%) of childhood cancers. It was first described by James Ewing in $1921,{ }^{5}$ as a diffuse endothelioma. Immunocytochemical, cytogenetic, and molecular genetic investigations have suggested a neural crest origin for the ES family of tumors, but the source of Ewing cells is still controversial. ${ }^{6} \mathrm{ES}$ occurs most commonly in the second decade of life, and as with many pediatric tumors shows a slight male predominance, and the location is usually the shaft of long bones and the central axis. ${ }^{7}$ Molecular biology states that these tumours arise from common gene rearrangements involving EWS gene on chromosome 22, reciprocal translocation with FLI1 gene on chromosome $11 \mathrm{t}(11 ; 22$ (q24;q12) \{ Analysed by PCR and FISH ${ }^{8}{ }^{8}$

Ewing Sarcoma of the clavicle is an unusual lesion and there are few reports about the surgical management in the literature. The fibula, rib, and clavicle, have been traditionally called expendable bones, and most of the authors recommend resection with no need of reconstruction. ${ }^{8}$

Good functional results have been reported with this method. $^{3-4}$

Juan Rodriguez Martin et $\mathrm{al}^{9}$ reported 5 cases ( 3 males and 2females) of clavicle Ewing sarcoma in the year 2009 in Journal of Pediatric Hematological Oncology. They concluded that good shoulder function can be achieved with no need of reconstruction after total claviculectomy.

Venkatraman Radhakrishnan, Shishir Rastogi AND Sameer Bakhshi ${ }^{10}$ analyzed 4 patients with clavicular ES in the year 2010 and reviewed the literature on management of this rare tumor \& found that Clavicular ES in contrast to ES of other sites seems to have a female preponderance and outcomes are similar to non-metastatic ES of other common sites.

Yijun Zhou et al ${ }^{11}$ documented resection of the clavicle in a 35 years old adult with excellent residual function. The patient had good functional outcomes with no pain of the shoulder at the follow-up of ten months. Fabrizio Albarello ${ }^{12}$ reported a case of right sternoclavicular Ewing Sarcoma in the year 2015 .

Extent of resection of clavicle is dependent on the location, histopathological type and stage of the tumor according to new classification system evolved for clavicular resections. Mayil, Meller and Malawer ${ }^{13}$ classified clavicular resections as followed:

Type I Medial one third

Type II Lateral one third

Type III Intercalary resection

Type IV Total claviculectomy

Type $\mathbf{V}$ Extended claviculectomy (i.e. part of the acromion or upper sternum)

He confirmed that post-claviculectomy, functions and oncological results are good and cosmetically acceptable\& thus removal of clavicle is not a disaster; in fact, clavicle is an accessory baggage in the skeleton.
SG Krishnan et al ${ }^{14}$ conducted a retrospective review of the records of six patients who had undergone unilateral claviculectomy. The functional outcomes following claviculectomy were good in this group of six patients despite a high complication rate. He suggested total claviculectomy as an useful salvage procedure for clinical situations in which the restoration of normal clavicular osseous anatomy is impossible. Acceptable pain relief and few or no deficits in activities of daily living can be expected.

Santiago Argüelles, Martín Rodríguez, Enrique Pereira ${ }^{15}$ from literature analysis concluded that total claviculectomy is an option to be taken into account when therapeutic options have exhausted as in cases of infection, plexopathy or sarcoma.

Rubright $^{16}$ assessed five patients 5 and 10 years after total claviculectomy using the DASH (Disability of Arm, Shoulder and Hand), the SF-36 (Short-Form-36), the SST (Simple Shoulder Test), the ASES (American Shoulder and Elbow Surgeons), the UCLA (UCLA Shoulder Test), the HSS (Hospital for Special Surgery Rating Scale) and the Constant scores, along with isokinetic tests, range of motion tests and kinematic analyses. The author concluded that patients undergoing claviculectomy lose $33 \%$ of adduction strength, but were able to compensate it with minimal functional deficit.

In 2012, Oheim et al ${ }^{17}$ published a consecutive series of 5 claviculectomy patients with an average follow-up of 9.4 months. Constant scores improved significantly in all patients postoperatively from 82 to 95 .

From these studies, it would appear that claviculectomy usually does not lead to a significant deficit in shoulder function.

\section{Conclusion}

Total claviculectomy is a highly successful method employed for bone tumours with good oncological and functional results. Ewing's sarcoma is a highly aggressive tumour with distant metastasis being worst prognostic factor. Recent molecular investigations have allowed more accurate diagnosis of Ewing's sarcoma. Successful outcome in ewing sarcoma depends on prompt initiation of neo-adjuvant chemotherapy with addition of radiotherapy if required. We conclude that functional and oncological outcome after Total Clavicular Resection in Non -Metastatic Ewing sarcoma is excellent.

\section{Conflict of Interest: None.}

\section{References}

1. Campbell's operative orthopaedics $13^{\text {th }}$ edition Vol.1 Pg. 953

2. Li J, Wang Z, Fu J. Surgical treatment of clavicular malignancies. J Shoulder Elbow Surg 2011;20:295-300.

3. Lewis MM, Ballet FL, Kroll PG. En bloc clavicular resection: operative procedure and postoperative testing of function. Clin Orthop Relat Res 1985;193:214-20.

4. Wood VE. The result of total claviculectomy. Clin Orthop Relat Res 1986;207:186-90.

5. Wessel RN, Schaap GR. Outcome of total claviculectomy in six cases. J Shoulder Elbow Surg 2007;16:312-5. 
6. Ewing J. Diffuse endothelioma of bone. Proc N Y Pathol Soc 1921;21:17-24.

7. Ginsberg JP, Woo SY, Johnson ME. Ewing's sarcoma family of tumors: Ewing's sarcoma of bone and soft tissue and the peripheral primitive neuroectodermal tumors. In: Pizzo PA, Poplack DG, eds. Principles and Practice of Pediatric

8. Oncology. 4th ed. Philadelphia: Lippincott Williams \&Wilkins;2002:973-1015

9. Eliazer S, Spencer J, Ye D. Alteration of mesodermal cell differentiation by EWS/FLI-1, the oncogene implicated in Ewing's sarcoma. Mol Cell Biol 2003;23:482-92.

10. Ozaki T, Hillmann A, Lindner N. Surgical treatment of bone sarcomas of the fibula. Analysis of 19 cases. Arch Orthop Trauma Surg 1997;116:475-9.

11. Rodriguez Martin, J., Pretell Mazzini, J., Viña Fernandez, R., Marti Ciruelos, R., \& Curto de la Mano, A. (2009). Ewing Sarcoma of Clavicle in Children. J Pediatric Hematol/Oncol 2009;31(11):820-4. doi:10.1097/mph.0b013e3181b7896f

12. Radhakrishnan, V., Rastogi, S., \& Bakhshi, S. Ewing sarcoma of the clavicle: A case series. Indian Pediatr 2011;48(2):1334. doi:10.1007/s13312-011-0038-5

13. Yijun Zhou, Zheng Tian, Akbar Yunus, Jiangtao Chen, Chong Wang, Leilei Xu, Xinghua Song. A Case of Ewing's Sarcoma of the Clavicle in Adults. Int J Clin Med 2013;4:343-6. doi:10.4236/ijcm.2013.48060

14. Albarello F, Deriu G, Goletti S, Campioni P., Extraosseus Ewing sarcoma: An uncommon periclavicular location. Radiol Case Rep (Online) 2015;10(2);1117.
15. Natarajan M, Meller I, Malawar M M. Claviculectomy for bone tumors. Indian J Orthop [serial online] 2006 \{cited 2018 Dec 301];40;115-8. Available from: http://www.ijoonline.com/text.asp?2006/40/2/115/34454

16. Krishnan, S. G., Schiffern, S. C., Pennington, S. D., Rimlawi, M., \& Burkhead, W. Z. Functional Outcomes after total Claviculectomy as a Salvage Procedure. J Bone Joint Surg 2007;89(6):1215-9. doi:10.2106/jbjs.e.01436

17. Santiago Argüelles, Martín Rodríguez, Enrique Pereira. Total claviculectomy as a rescue procedure Case report and literature review. Rev Asoc Argent Ortop Traumatol 2018;83(2):124-30.

18. Rubright J, Kelleher P, Beardsley C, Paller D, Shackford S, Beynnon B, et al. Long term clinical outcomes, motion, strength, and function after total claviculectomy. J Shoulder Elbow Surg 2014;23(2)236-44.

19. Oheim R, Schulz AP, Schoop R, Grimme CH, Gille J, Gerlach $\mathrm{U}$. Medium-term results after total clavicle resection in cases of osteitis: a consecutive case series of five patients. Int Orthop 2012;36:775-81. http://dx.doi.org/10.1007/s00264-011-1342-3

How to cite this article: Mallik M, Nayak SS, Lenka S, Mishra NR, Das A. Total claviculectomy in Ewing's sarcoma clavicle: A rare case report and review of literature. Indian J Orthop Rheumatol 2019;5(1):168-71. 